\title{
藤黄酸衍生物的合成及抗肿瘤活性研究
}

\author{
张生烈 $a, b$ 李 乾 ${ }^{a, b}$ 张 䂞 ${ }^{a, b}$ 孙昊鹏*,a,b 尤启冬*,a,b,c \\ $\left({ }^{a}\right.$ 天然药物活性组分与药效国家重点实验室 中国药科大学 南京 210009) \\ ( ${ }^{b}$ 中国药科大学药物化学教研室 南京 210009) \\ ( ${ }^{c}$ 江苏省肿瘤发生与干预重点实验室 中国药科大学 南京 210009)
}

\begin{abstract}
摘要 以藤黄酸为原料, 经过 C-32 位选择性环氧化、高碘酸氧化、甲基化、Jones 氧化、不同条件的酯化、酰胺化等 反应步骤, 合成了 17 个藤黄酸衍生物. 所有目标化合物的结构均通过核磁共振谱、红外光谱和质谱证实, 并采用 MTT 比色法对所合成的目标化合物进行了体外抗肿瘤细胞生物活性测试. 结果表明, 化合物 $5,7,8,13$ 对人肝癌细胞 (HepG2)、人结肠癌细胞(HCT-116)的增殖抑制活性显著强于藤黄酸.
\end{abstract}

关键词 藤黄酸; 衍生物; 合成; 抗肿瘤; 生物活性

\section{Synthesis and Bioevaluation of Gambogic Acid Derivatives as Antitumor Agents}

\author{
Zhang, Shenglie $\mathrm{L}^{a, b} \quad$ Li, Qian ${ }^{a, b} \quad$ Zhang, Lei ${ }^{a, b} \quad$ Sun, Haopeng ${ }^{*, a, b} \quad$ You, Qidong ${ }^{*, a, b, c}$ \\ ( ${ }^{a}$ State Key Laboratory of Natural Medicines, China Pharmaceutical University, Nanjing 210009) \\ ( ${ }^{b}$ Department of Medicinal Chemstry, China Pharmaceutical University, Nanjing 210009) \\ ( ${ }^{c}$ Jiangsu Key Laboratory of Carcinogenesis and Intervention, China Pharmaceutical University, Nanjing 210009)
}

\begin{abstract}
Seventeen new compounds were synthesized from gambogic acid (GA) with the structural modification of C-32. The reaction steps included selective epoxidation, periodate oxidation, methylation, Jones oxidation, different conditions of esterification, amidation, etc. The structures of target compounds were confirmed by NMR, IR and MS/ESI techniques. Their antitumor activities were evaluated in vitro by MTT assay. The results showed that compounds 5, 7, 8 and 13 were more potent than GA to human hepatoma cells (HepG2) and colon carcinoma cells (HCT-116).

Keywords gambogic acid; derivative; synthesis; antitumor; biological activity
\end{abstract}

藤黄酸(gambogic acid, GA)具有良好的抗肿瘤活性 和细胞选择性, 一直以来为人们所重视 ${ }^{[1 \sim 3]}$. 为了寻找 活性更优的藤黄酸衍生物, 人们对藤黄酸进行了化学结 构修饰研究. 目前有关藤黄酸衍生物的报道中, 对其结 构修饰主要集中在 C-6 酚差基、C-8 羰基、C-9, C-10 位 双键、C-30 羧基、C-32, C-33 双键和 C-37, C-38 双键上 (图 1).

Zhang 等 ${ }^{[4]}$ 研究发现藤黄酸结构中 C-9, C-10 位的双 键对于藤黄酸诱导细胞调亡起到了重要作用, 当双键被 还原后，抗肿瘤活性大大降低; 而 C-6 位的酚羟基和 C-30 位的羧基能够进行多种修饰, 并保持与 GA 相似的

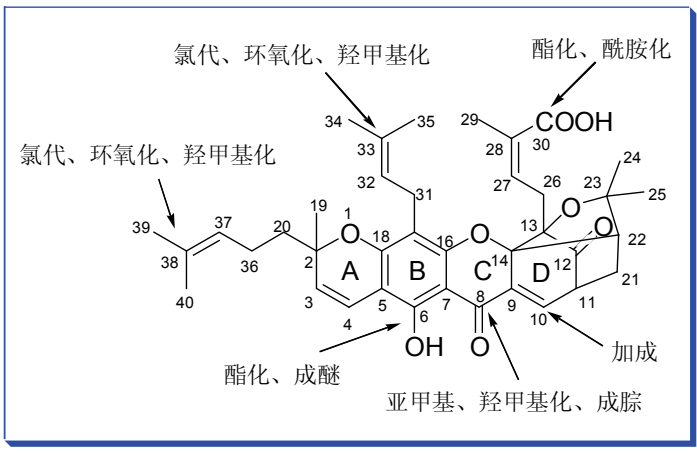

图 1 藤黄酸结构修饰的位点

Figure 1 Structural modification of gambogic acid

\footnotetext{
*E-mail: youqidong@gmail.com; sunhaopeng@163.com

Received April 18, 2012; revised May 9, 2012; published online May 11, 2012.

Project supported by the National Natural Science Foundation of China (No. 90713038) and the National Key Program for New Drug Development (No. 2009ZX09501-003).

国家自然科学基金(No. 90713038)和重大新药创制科技重大专项(No. 2009ZX09501-003)资助项目.
} 
抗肿瘤活性. $\mathrm{He}$ 等 $^{[5]}$ 对 $\mathrm{GA}$ 的 C-30 位的羧基进行酯化, 得到一系列 GA 衍生物, 不但改善了水溶性, 一些衍生 物还显示出比 GA 更好的生物活性. 近年来, 我们课题 组也在 $\mathrm{GA}$ 衍生物的设计合成与生物活性研究方面做了 一些工作: Feng 等 ${ }^{[6]}$ 合成的化合物 33 -氯化转位藤黄酸 和 33,37-二氯转位藤黄酸抗肿瘤活性优于 GA; Wang 等 ${ }^{[7]}$ 对其进一步研究, 发现了更具潜力的先导物 38,40环氧化-33-氯化转位藤黄酸, 并对构效关系做了更深入 的探讨; Wang 等 ${ }^{[8]}$ 对 GA 衍生物的研究中发现, 针对 C-35, C-39 位甲基修饰, C-32, C-33 和 C-37, C-38 双键改 造, 可以提高 $\mathrm{GA}$ 抗肿瘤活性; Wang 等 ${ }^{[9]}$ 以 $\mathrm{GA}$ 为原料, 通过微波方法合成了两个新化合物, 活性与 GA 相当, 该合成方法简单、迅速、收率高, 为 GA 衍生物的合成 制备提供了新的有效途径和方法.

为了更深入地研究藤黄酸 $\mathrm{B}$ 环的异戊烯基侧链, 结 合上述的研究成果, 我们重新设计了一系列的 GA 衍生 物, 以期得到结构更优化、疗效更显著、特异性更好的 先导化合物, 并进一步研究 GA 的构效关系. 本文通过 在侧链位置引入不同取代基来考察其对藤黄酸抗肿瘤 活性的影响. 具体思路是以藤黄酸为先导物, 在藤黄酸 32 位碳原子 (C-32)上进行结构修饰, 经过 C-32 选择性环 氧化、高碘酸断键得醛、甲基化、Jones 氧化、不同条 件的酯化、酰胺化等反应步骤, 合成了 17 个藤黄酸衍生 物. 合成路线见 Scheme 1.

\section{1 实验部分}

\section{1 仪器与试剂}

Bruker ARX-300 型核磁共振仪 $\left(\mathrm{CDCl}_{3}\right.$ 或 DMSO- $d_{6}$ 为溶剂, TMS 为内标); Nicolet Impact 410 型傅立叶变换 红外光谱测定 ( KBr 压片); Agilent 1946A-MSD 型质谱仪 (ESIMS); Q-Tof micro 四极杆飞行时间串联质谱仪 (HRMS). 实验所用试剂均为分析纯; 藤黄酸原料自制, 纯度大于 $95 \%$ (HPLC).

\section{$1.232,33$-环氧一藤黄酸(2)的合成}

在 $0{ }^{\circ} \mathrm{C}$ 下往 $50 \mathrm{~mL}$ 的烧瓶中加入 $20 \mathrm{~mL}$ 的 $\mathrm{CH}_{2} \mathrm{Cl}_{2}$, 然后加入 $500 \mathrm{mg}(0.80 \mathrm{mmol})$ 的藤黄酸 $(1)$ 及 $165 \mathrm{mg}$ (0.96 mmol)的间氯过氧苯甲酸, 而后自然升温, 室温下 搅拌 $8 \mathrm{~h}$, 浓缩, 柱层析 $[V$ (石油醚) $: V($ 乙酸乙酯 $)=4$ : 1]洗脱, 得黄色粘稠物 $180 \mathrm{mg}$, 收率为 $35 \% .{ }^{1} \mathrm{H}$ NMR $\left(\mathrm{CDCl}_{3}, 300 \mathrm{MHz}\right) \delta: 1.27$ (s, 3H, 19-H), 1.29 1.33 (m, $3 \mathrm{H}, 24-\mathrm{H}), 1.35 \sim 1.38$ (m, 1H, 21b-H), 1.41 (s, 3H, 40-H), 1.48 (s, 3H, 35-H), 1.63 (s, 3H, 39-H), 1.66 (s, 2H, 20-H), 1.69 (s, 3H, 29-H), 1.76 (s, 3H, 25-H), 1.94 (s, 3H, 34-H), $2.05 \sim 2.07(\mathrm{~m}, 2 \mathrm{H}, 36-\mathrm{H}), 2.30 \sim 2.32(\mathrm{~m}, 1 \mathrm{H}, 21 \mathrm{a}-\mathrm{H})$, $2.52(\mathrm{~d}, J=9.0 \mathrm{~Hz}, 1 \mathrm{H}, 22-\mathrm{H}), 2.81 \sim 2.84(\mathrm{~m}, 1 \mathrm{H}, 32-\mathrm{H})$,
2.97 (d, $J=7.2 \mathrm{~Hz}, 2 \mathrm{H}, 26-\mathrm{H}), 3.13 \sim 3.17(\mathrm{~m}, 1 \mathrm{H}, 31 \mathrm{~b}-\mathrm{H})$, $3.24 \sim 3.27(\mathrm{~m}, 1 \mathrm{H}, 31 \mathrm{a}-\mathrm{H}), 3.50 \sim 3.51(\mathrm{~m}, 1 \mathrm{H}, 11-\mathrm{H})$, $5.08 \sim 5.10$ (m, $1 \mathrm{H}, 37-\mathrm{H}), 5.36(\mathrm{~d}, J=10.2 \mathrm{~Hz}, 1 \mathrm{H}, 3-\mathrm{H})$, $6.13 \sim 6.17(\mathrm{~m}, 1 \mathrm{H}, 27-\mathrm{H}), 6.62(\mathrm{~d}, J=10.2 \mathrm{~Hz}, 1 \mathrm{H}, 4-\mathrm{H})$, $7.56(\mathrm{~d}, J=6.9 \mathrm{~Hz}, 1 \mathrm{H}, 10-\mathrm{H}), 12.76(\mathrm{~s}, 1 \mathrm{H}, 6-\mathrm{OH}) ;{ }^{13} \mathrm{C}$ NMR (75 MHz, $\left.\mathrm{CDCl}_{3}\right) \delta$ : 17.6 (C-40), 18.1 (C-34), 20.3 (C-29), 21.6 (C-36), 25.0 (C-21), 25.1 (C-35), 25.8 (C-39), 27.2 (C-36), 27.5 (C-19), 28.9 (C-24), 29.4 (C-26), 30.3 (C-25), 42.2 (C-20), 42.9 (C-33), 44.7 (C-32), 46.3 (C-11), 48.8 (C-22), 79.7 (C-2), 83.1 (C-13), 83.3 (C-23), 86.0 (C-14), 90.4 (C-7), 98.0 (C-5), 106.9 (C-17), 116.5 (C-4), 121.1 (C-37), 126.1 (C-3), 127.1 (C-28), 131.6 (C-38), 133.2 (C-9), 135.2 (C-10), 135.7 (C-27), 158.0 (C-16), 159.2 (C-6), 166.8 (C-18), 171.6 (C-30), 174.1 (C-8), 203.5 (C-12); IR (film) v: 3428, 2927, 1740, 1713, 1688, 1628, 1436, 1188, $884 \mathrm{~cm}^{-1}$; ESI-MS $m / z: 645[\mathrm{M}+\mathrm{H}]^{+}$.

\subsection{7-去异戊烯-17-甲酰基甲基-藤黄酸(3)的合成}

将化合物 2 (100 mg, $0.155 \mathrm{mmol})$ 溶于 $10 \mathrm{~mL}$ 四氢呋 喃中, $0{ }^{\circ} \mathrm{C}$ 下加入 $\mathrm{HIO}_{4} \cdot 2 \mathrm{H}_{2} \mathrm{O} 40 \mathrm{mg}(0.175 \mathrm{mmol})$, 摚 拌, 反应液呈淡黄色透明, 室温反应 $1 \mathrm{~h}$ 后, 加少量水至 反应液中, 乙酸乙酯萃取, 分出乙酸乙酯层, 用饱和食 盐水洗, 无水硫酸镁干燥, 蒸出溶剂, 柱层析 $[V$ (石油 醚) $: V($ 乙酸乙酯 $)=2: 1$ ]洗脱, 得黄色粘稠物 $22 \mathrm{mg}$, 收率 24\%. ${ }^{1} \mathrm{H}$ NMR $\left(\mathrm{CDCl}_{3}, 300 \mathrm{MHz}\right) \delta: 1.28(\mathrm{~s}, 3 \mathrm{H}$, $19-\mathrm{H}), 1.39 \sim 1.43(\mathrm{~m}, 1 \mathrm{H}, 21 \mathrm{~b}-\mathrm{H}), 1.45 \sim 1.48(\mathrm{~m}, 3 \mathrm{H}$, 24-H), $1.64 \sim 1.67(\mathrm{~m}, 6 \mathrm{H}, 29-\mathrm{H}, 40-\mathrm{H}), 1.69$ (s, 3H, $39-\mathrm{H}), 1.79 \sim 1.82(\mathrm{~m}, 5 \mathrm{H}, 20-\mathrm{H}, 25-\mathrm{H}), 2.10 \sim 2.12(\mathrm{~m}$, $2 \mathrm{H}, 36-\mathrm{H}), 2.31 \sim 2.35(\mathrm{~m}, 1 \mathrm{H}, 21 \mathrm{a}-\mathrm{H}), 2.51(\mathrm{~d}, J=9.0 \mathrm{~Hz}$, $1 \mathrm{H}, 22-\mathrm{H}), 2.95 \sim 2.97(\mathrm{~m}, 2 \mathrm{H}, 26-\mathrm{H}), 3.30(\mathrm{~d}, J=7.6 \mathrm{~Hz}$, $1 \mathrm{H}, 31 \mathrm{~b}-\mathrm{H}), 3.40 \sim 3.42(\mathrm{~m}, 2 \mathrm{H}, 31 \mathrm{a}-\mathrm{H}, 11-\mathrm{H}), 5.20 \sim 5.23$ (m, $1 \mathrm{H}, 37-\mathrm{H}), 5.65(\mathrm{~d}, J=10.2 \mathrm{~Hz}, 1 \mathrm{H}, 3-\mathrm{H}), 5.92 \sim 5.97$ (m, 1H, 27-H), 6.57 (d, $J=10.1 \mathrm{~Hz}, 1 \mathrm{H}, 4-\mathrm{H}), 7.51$ (d, $J=$ $6.9 \mathrm{~Hz}, 1 \mathrm{H}, 10-\mathrm{H}), 9.62(\mathrm{~s}, 1 \mathrm{H}, \mathrm{CHO}), 12.74(\mathrm{~s}, 1 \mathrm{H}$, 6-OH); IR (KBr) v: 3408, 2935, 1716, 1630, 1587, 1452, 1425, 1380, 1296, 1229, 1129, $789 \mathrm{~cm}^{-1}$; ESI-MS m/z: 603 $[\mathrm{M}+\mathrm{H}]^{+}$.

\subsection{7-去异戊烯-17-甲酰基甲基-6-甲氧基藤黄酸甲 酯(4)的合成}

将化合物 3 (50 mg, $0.083 \mathrm{mmol}$ )溶于 $10 \mathrm{~mL}$ 丙酮中, 加入无水 $\mathrm{K}_{2} \mathrm{CO}_{3} 115 \mathrm{mg}(0.83 \mathrm{mmol}), \mathrm{N}_{2}$ 保护下室温搅 拌, 约 $30 \mathrm{~min}$ 后滴加 $0.1 \mathrm{~mL}(2.0 \mathrm{mmol}) \mathrm{CH}_{3} \mathrm{I}$, 室温下搅 拌反应 $24 \mathrm{~h}$. 反应完毕后, 过滤除去 $\mathrm{K}_{2} \mathrm{CO}_{3}$, 浓缩, 柱层 析 $[V($ 石油醚 $): V($ 乙酸乙酯 $)=4: 1]$ 洗脱, 得淡黄色粘 稠物 $50 \mathrm{mg}$, 收率为 $96 \% .{ }^{1} \mathrm{H} \mathrm{NMR}\left(\mathrm{CDCl}_{3}, 300 \mathrm{MHz}\right) \delta$ : 


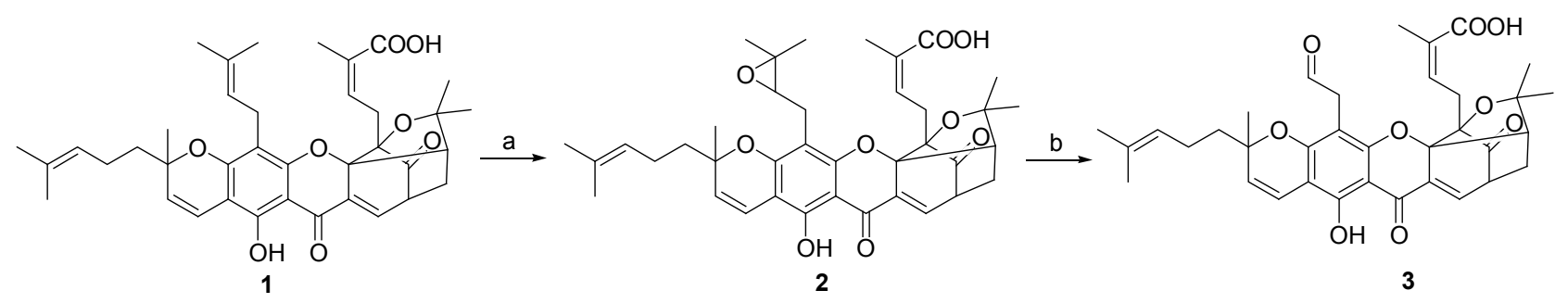

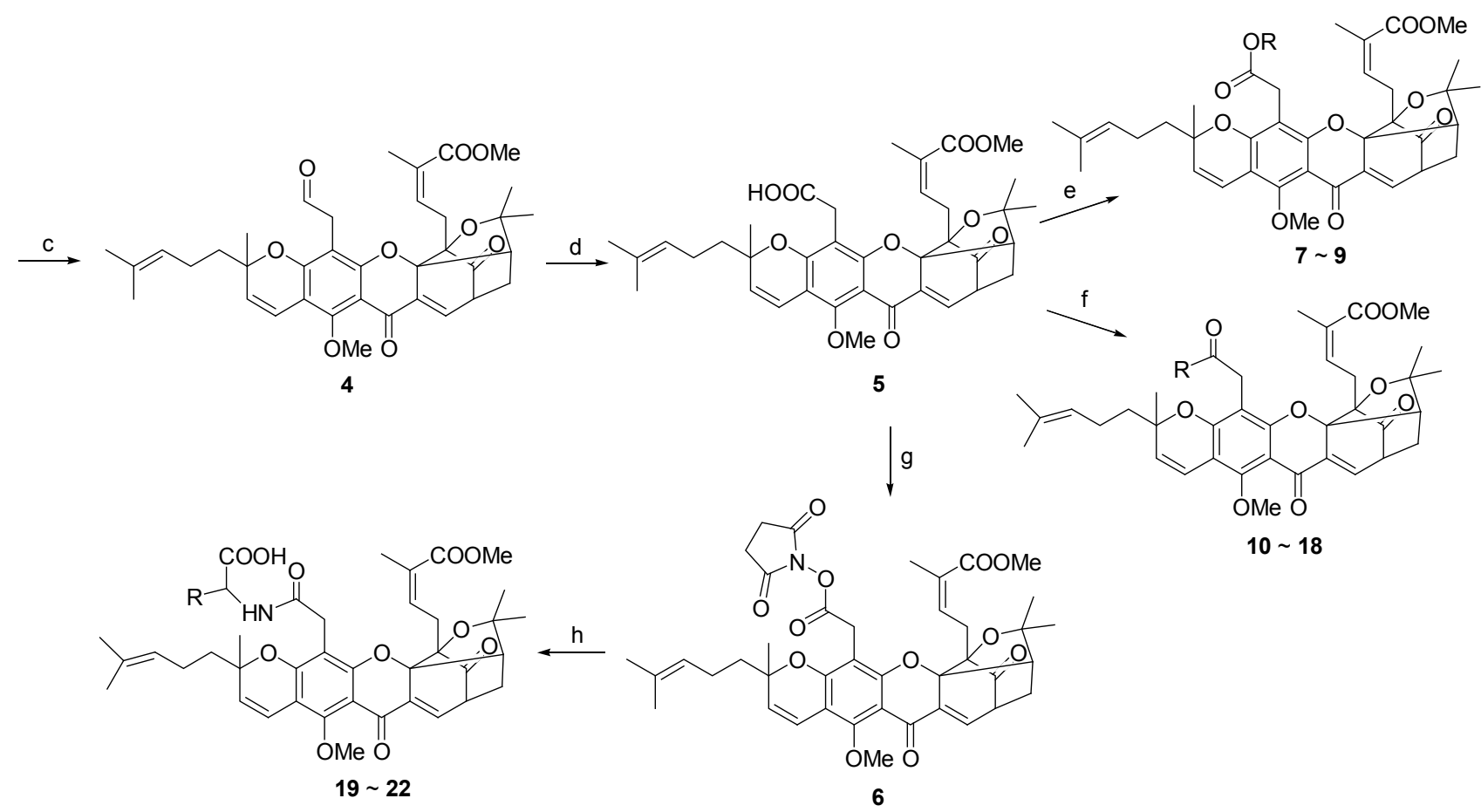<smiles>[3H][Fe]CC</smiles>

$8 \mathrm{R}=\mathrm{CH}_{\mathrm{CH}_{3}}^{\mathrm{CH}_{3}}$<smiles>[R]N1CCCCC1</smiles>
$12 \mathrm{R}=-\mathrm{N}$ $9 \mathrm{R}=\frac{\mathrm{CH}_{3}}{\mathrm{CH}_{3}}$ $10 \mathrm{R}=-\mathrm{N} \mathrm{O}$<smiles>[13B]=CNc1ccc(C)cc1</smiles><smiles>[R]CNc1ccc(OC)cc1</smiles>

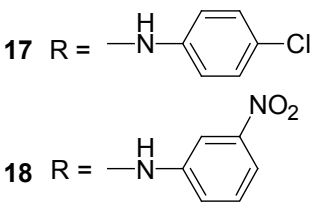

$19 \mathrm{R}=-\mathrm{H}$<smiles>CC(C)[12PH]</smiles>

$21 \mathrm{R}=\widehat{\mathrm{S}}-\mathrm{CH}_{3}$

$22 \mathrm{R}=\widehat{\mathrm{SH}}_{\mathrm{SH}}$

Reagents and conditions: (a) $m$-CPBA, $\mathrm{CH}_{2} \mathrm{Cl}_{2}, 0{ }^{\circ} \mathrm{C}, 35 \%$; (b) $\mathrm{HIO}_{4} \cdot 2 \mathrm{H}_{2} \mathrm{O}$, THF, r.t., $24 \%$; (c) $\mathrm{CH}_{3} \mathrm{l}, \mathrm{K}_{2} \mathrm{CO}_{3}, \mathrm{CH}_{3} \mathrm{COCH}_{3}$, r.t., $96 \%$; (d) Jones reagent, $\mathrm{CH}_{3} \mathrm{COCH}_{3}, 68 \%$; (e) DMAP, EDCl, EtOH, 35\% 43\%; (f) DMAP, EDCl, THF, 23\% 43\%; (g) NHS, DCC, $\mathrm{CH}_{2} \mathrm{Cl}_{2}, 43 \%$; (h) Amino acids, $\mathrm{Et}_{3} \mathrm{~N}, \mathrm{DMSO}, 23 \% \sim 33 \%$

\section{Scheme 1}

$1.28(\mathrm{~s}, 3 \mathrm{H}, 19-\mathrm{H}), 1.39 \sim 1.43(\mathrm{~m}, 1 \mathrm{H}, 21 \mathrm{~b}-\mathrm{H}), 1.46 \sim 1.51$ (m, 3H, 24-H), 1.64 (s, 6H, 29-H, 40-H), 1.69 (s, 3H, 39-H), $1.78 \sim 1.81(\mathrm{~m}, 5 \mathrm{H}, 20-\mathrm{H}, 25-\mathrm{H}), 2.31$ (d, $J=4.8$ $\mathrm{Hz}, 1 \mathrm{H}, 21 \mathrm{a}-\mathrm{H}), 2.51$ (d, $J=9.0 \mathrm{~Hz}, 1 \mathrm{H}, 22-\mathrm{H}), 2.58 \sim 2.61$ (m, 2H, 36-H), 2.93 (d, J=7.23 Hz, 2H, 26-H), 3.10 (d, $J=7.6 \mathrm{~Hz}, 1 \mathrm{H}, 31 \mathrm{~b}-\mathrm{H}), 3.33 \sim 3.36(\mathrm{~m}, 1 \mathrm{H}, 31 \mathrm{a}-\mathrm{H}), 3.41$ (s, $\left.3 \mathrm{H}, \mathrm{COOCH}_{3}\right), 3.43 \sim 3.47(\mathrm{~m}, 1 \mathrm{H}, 11-\mathrm{H}), 3.82(\mathrm{~s}, 3 \mathrm{H}$, 6- $\left.\mathrm{OCH}_{3}\right), 5.05 \sim 5.07(\mathrm{~m}, 1 \mathrm{H}, 37-\mathrm{H}), 5.56(\mathrm{~d}, J=10.2 \mathrm{~Hz}$, $1 \mathrm{H}, 3-\mathrm{H}), 6.06 \sim 6.08(\mathrm{~m}, 1 \mathrm{H}, 27-\mathrm{H}), 6.65(\mathrm{~d}, J=10.7 \mathrm{~Hz}$,
1H, 4-H), $7.51(\mathrm{~d}, J=6.8 \mathrm{~Hz}, 1 \mathrm{H}, 10-\mathrm{H}), 9.60(\mathrm{~s}, 1 \mathrm{H}$, CHO); IR (KBr) v: 2971, 1736, 1718, 1710, 1673, 1464, 1383, 1166, $1020 \mathrm{~cm}^{-1}$; ESI-MS m/z: $631[\mathrm{M}+\mathrm{H}]^{+}$.

\subsection{7-去异戊烯-17-羧基甲基-6-甲氧基藤黄酸甲酯} (5)的合成

将化合物 4 (20 mg, $0.032 \mathrm{mmol}$ )溶于丙酮 $5 \mathrm{~mL}$ 中, 0 ${ }^{\circ} \mathrm{C}$ 下滴加 Jones 试剂 $(2.5 \mathrm{~mol} / \mathrm{L}) 0.015 \mathrm{~mL}(0.038 \mathrm{mmol})$, 反应液呈绿色混浊, 反应 $0.5 \mathrm{~h}$ 后停止反应, 加少量饱和 碳酸氢钠水溶液至反应液中, 乙酸乙酯萃取, 分出乙酸 
乙酯层, 用饱和食盐水洗, 无水硫酸钠干燥, 蒸出溶剂, 柱层析 $[V$ (石油醚) $: V($ 乙酸乙酯 $)=1: 1$ ] 洗脱, 得黄色 粘稠物 $14 \mathrm{mg}$, 收率 $68 \% .{ }^{1} \mathrm{H}$ NMR $\left(\mathrm{CDCl}_{3}, 300 \mathrm{MHz}\right) \delta$ : 1.26 (s, 3H, 24-H), 1.29 (s, 3H, 19-H), $1.38 \sim 1.40$ (m, $1 \mathrm{H}$, 21b-H), 1.48 (s, 3H, 40-H), 1.65 (s, 3H, 39-H), 1.68 (s, 2H, 20-H), 1.69 (s, 3H, 29-H), 1.76 (s, 3H, 25-H), 2.07 2.11 (m, 2H, 36-H), $2.18(\mathrm{~s}, 1 \mathrm{H}, 21 \mathrm{a}-\mathrm{H}), 2.52(\mathrm{~d}, J=9.3 \mathrm{~Hz}$, $1 \mathrm{H}, 22-\mathrm{H}), 2.96 \sim 2.98(\mathrm{~m}, 2 \mathrm{H}, 26-\mathrm{H}), 3.21 \sim 3.25(\mathrm{~m}, 1 \mathrm{H}$, $31 \mathrm{a}-\mathrm{H}), 3.26 \sim 3.28(\mathrm{~m}, 1 \mathrm{H}, 31 \mathrm{~b}-\mathrm{H}), 3.39(\mathrm{~s}, 1 \mathrm{H}, 11-\mathrm{H})$, $3.42\left(\mathrm{~s}, 3 \mathrm{H}, 6-\mathrm{OCH}_{3}\right), 3.81\left(\mathrm{~s}, 3 \mathrm{H}, \mathrm{COOCH}_{3}\right), 5.06 \sim 5.08$ (m, 1H, 37-H), $5.50(\mathrm{~d}, J=10.2 \mathrm{~Hz}, 1 \mathrm{H}, 3-\mathrm{H}), 5.95 \sim 5.98$ (m, 1H, 27-H), 6.71 (d, $J=10.2 \mathrm{~Hz}, 1 \mathrm{H}, 4-\mathrm{H}), 7.45$ (d, $J=$ $6.9 \mathrm{~Hz}, 1 \mathrm{H}, 10-\mathrm{H})$; IR (KBr) v: 3408, 2935, 1716, 1630, 1587, 1452, 1425, 1380, 1296, 1229, 1129, $789 \mathrm{~cm}^{-1}$; ESI-MS $m / z$ : $646[\mathrm{M}-\mathrm{H}]^{-}$; HRMS calcd for $\mathrm{C}_{37} \mathrm{H}_{43} \mathrm{O}_{10}$ $(\mathrm{M}+\mathrm{H})^{+}$647.2851, found 647.2835.

\subsection{7-去异戊烯-17-乙氧基甲酰基甲基-6-甲氧基-藤 黄酸甲酯(7)的合成}

将化合物 5 (40 mg, $0.062 \mathrm{mmol}$ ) 溶于 $5 \mathrm{~mL}$ 乙醇中, 加入 DMAP $9 \mathrm{mg}(0.074 \mathrm{mmol})$ 和 EDCI $14 \mathrm{mg}(0.074$ $\mathrm{mmol}$ ) 后室温搅拌反应 $8 \mathrm{~h}$, 加入饱和 $\mathrm{NH}_{4} \mathrm{Cl}$ 水溶液并摚 拌 $5 \mathrm{~min}$, 乙酸乙酯萃取, 分出乙酸乙酯层, 饱和食盐水 洗, 无水硫酸钠干燥, 蒸出溶剂, 柱层析 $[V$ (石油醚) : $V($ 乙酸乙酯 $)=4: 1]$ 洗脱, 得黄色油状物 $18 \mathrm{mg}$, 收率 $43 \% .{ }^{1} \mathrm{H}$ NMR $\left(\mathrm{CDCl}_{3}, 300 \mathrm{MHz}\right) \delta: 1.24 \sim 1.25(\mathrm{~m}, 3 \mathrm{H}$, $\left.\mathrm{OCH}_{2} \mathrm{CH}_{3}\right), 1.26 \sim 1.28(\mathrm{~m}, 3 \mathrm{H}, 24-\mathrm{H}), 1.29(\mathrm{~s}, 3 \mathrm{H}, 19-\mathrm{H})$, $1.38 \sim 1.40(\mathrm{~m}, 1 \mathrm{H}, 21 \mathrm{~b}-\mathrm{H}), 1.48(\mathrm{~s}, 3 \mathrm{H}, 40-\mathrm{H}), 1.65$ (s, $3 \mathrm{H}, 39-\mathrm{H}), 1.68$ (s, 2H, 20-H), 1.69 (s, 3H, 29-H), 1.76 (s, $3 \mathrm{H}, 25-\mathrm{H}), 2.08 \sim 2.10(\mathrm{~m}, 2 \mathrm{H}, 36-\mathrm{H}), 2.18(\mathrm{~s}, 1 \mathrm{H}, 21 \mathrm{a}-\mathrm{H})$, $2.52(\mathrm{~d}, J=9.0 \mathrm{~Hz}, 1 \mathrm{H}, 22-\mathrm{H}), 2.96 \sim 2.98(\mathrm{~m}, 2 \mathrm{H}, 26-\mathrm{H})$, $3.20 \sim 3.22(\mathrm{~m}, 1 \mathrm{H}, 31 \mathrm{a}-\mathrm{H}), 3.25 \sim 3.29(\mathrm{~m}, 1 \mathrm{H}, 31 \mathrm{~b}-\mathrm{H})$, $3.43\left(\mathrm{~s}, 3 \mathrm{H}, 6-\mathrm{OCH}_{3}\right), 3.44(\mathrm{~s}, 1 \mathrm{H}, 11-\mathrm{H}), 3.81(\mathrm{~s}, 3 \mathrm{H}$, $\left.\mathrm{COOCH}_{3}\right), 4.11 \sim 5.13\left(\mathrm{~m}, 2 \mathrm{H}, \mathrm{OCH}_{2} \mathrm{CH}_{3}\right), 5.04 \sim 5.08$ (m, 1H, 37-H), 5.50 (d, $J=10.2 \mathrm{~Hz}, 1 \mathrm{H}, 3-\mathrm{H}), 5.92 \sim 5.95$ (m, 1H, 27-H), 6.69 (d, $J=10.2 \mathrm{~Hz}, 1 \mathrm{H}, 4-\mathrm{H}), 7.44$ (d, $J=$ $6.9 \mathrm{~Hz}, 1 \mathrm{H}, 10-\mathrm{H})$; IR (KBr) v: 3383, 2928, 2863, 2714, 2567, 2393, 2215, 1772, 1715, 1564, 1586, 1454, 1425, 1380, 1225, 1126, 1030, $752 \mathrm{~cm}^{-1}$; ESI-MS m/z: $675[\mathrm{M}+$ $\mathrm{H}]^{+}$; HRMS calcd for $\mathrm{C}_{39} \mathrm{H}_{47} \mathrm{O}_{10}(\mathrm{M}+\mathrm{H})^{+}$675.3164, found 675.3152 .

\subsection{7-去异戊烯-17-异丙氧基甲酰基甲基-6-甲氧基-} 藤黄酸甲酯(8)的合成

8 的合成方法同 7, 原料为 $\mathbf{5}$ 和异丙醇, 得到黄色油 状物 $18 \mathrm{mg}$, 收率 $42 \% .{ }^{1} \mathrm{H}$ NMR $\left(\mathrm{CDCl}_{3}, 300 \mathrm{MHz}\right) \delta$ : $1.15 \sim 1.18\left[\mathrm{~m}, 6 \mathrm{H}, \mathrm{OCH}\left(\mathrm{CH}_{3}\right)_{2}\right], 1.19$ (s, 3H, 19-H),
$1.21 \sim 1.23(\mathrm{~m}, 3 \mathrm{H}, 24-\mathrm{H}), 1.32 \sim 1.34(\mathrm{~m}, 1 \mathrm{H}, 21 \mathrm{~b}-\mathrm{H})$, 1.38 (s, 3H, 40-H), 1.54 (s, 3H, 39-H), 1.59 (s, 2H, 20-H), $1.62(\mathrm{~s}, 3 \mathrm{H}, 29-\mathrm{H}), 1.69$ (s, 3H, 25-H), 1.94 1.97 (m, 2H, $36-\mathrm{H}), 2.11$ (s, $1 \mathrm{H}, 21 \mathrm{a}-\mathrm{H}), 2.45$ (d, $J=9.3 \mathrm{~Hz}, 1 \mathrm{H}, 22-\mathrm{H})$, $2.89 \sim 2.91(\mathrm{~m}, 2 \mathrm{H}, 26-\mathrm{H}), 3.15 \sim 3.18(\mathrm{~m}, 1 \mathrm{H}, 31 \mathrm{a}-\mathrm{H})$, $3.28 \sim 3.30$ (m, $1 \mathrm{H}, 31 \mathrm{~b}-\mathrm{H}), 3.36\left(\mathrm{~s}, 3 \mathrm{H}, 6-\mathrm{OCH}_{3}\right), 3.40$ (s, $1 \mathrm{H}, 11-\mathrm{H}), 3.74\left(\mathrm{~s}, 3 \mathrm{H}, \mathrm{COOCH}_{3}\right), 4.90 \sim 4.92[\mathrm{~m}, 1 \mathrm{H}$, $\left.\mathrm{OCH}\left(\mathrm{CH}_{3}\right)_{2}\right], 4.95 \sim 4.97(\mathrm{~m}, 1 \mathrm{H}, 37-\mathrm{H}), 5.43$ (d, $J=10.2$ $\mathrm{Hz}, 1 \mathrm{H}, 3-\mathrm{H}), 5.83 \sim 5.85(\mathrm{~m}, 1 \mathrm{H}, 27-\mathrm{H}), 6.62(\mathrm{~d}, J=10.2$ $\mathrm{Hz}, 1 \mathrm{H}, 4-\mathrm{H}), 7.37$ (d, $J=6.9 \mathrm{~Hz}, 1 \mathrm{H}, 10-\mathrm{H})$; IR (KBr) $v$ : 3404, 2932, 2866, 2564, 2469, 2394, 2296, 2115, 1775, 1711, 1658, 1587, 1452, 1420, 1381, 1266, 1081, 1030, $754 \mathrm{~cm}^{-1}$; ESI-MS m/z: $689[\mathrm{M}+\mathrm{H}]^{+}$; HRMS calcd for $\mathrm{C}_{40} \mathrm{H}_{49} \mathrm{O}_{10}(\mathrm{M}+\mathrm{H})^{+} \quad 689.3326$, found 689.3312 .

\subsection{7-去异戊烯-17-叔丁氧基甲酰基甲基-6-甲氧基- 藤黄酸甲酯 $(9)$ 的合成}

9 的合成方法同 7, 原料为 $\mathbf{5}$ 和叔丁醇, 得到黄色油 状物 $15 \mathrm{mg}$, 收率 $35 \% .{ }^{1} \mathrm{H}$ NMR $\left(\mathrm{CDCl}_{3}, 300 \mathrm{MHz}\right) \delta$ : 1.17 (s, 3H, 19-H), $1.18 \sim 1.20$ (m, 3H, 24-H), 1.36 [s, 9H, $\left.\mathrm{OC}\left(\mathrm{CH}_{3}\right)_{3}\right], 1.35 \sim 1.37(\mathrm{~m}, 1 \mathrm{H}, 21 \mathrm{~b}-\mathrm{H}), 1.40(\mathrm{~s}, 3 \mathrm{H}$, 40-H), 1.50 (s, 3H, 39-H), 1.61 (s, 2H, 20-H), 1.63 (s, 3H, 29-H), 1.70 (s, 3H, 25-H), $1.90 \sim 1.93$ (m, 2H, 36-H), 2.10 (s, 1H, 21a-H), 2.44 (d, $J=9.3 \mathrm{~Hz}, 1 \mathrm{H}, 22-\mathrm{H}), 2.89 \sim 2.91$ (m, 2H, 26-H), 3.12 3.15 (m, 1H, 31a-H), 3.26 28 (m, $1 \mathrm{H}, 31 \mathrm{~b}-\mathrm{H}), 3.36$ (s, 3H, 6- $\left.\mathrm{OCH}_{3}\right), 3.52(\mathrm{~s}, 1 \mathrm{H}, 11-\mathrm{H}), 3.74$ (s, $\left.3 \mathrm{H}, \mathrm{COOCH}_{3}\right), 4.97 \sim 5.01(\mathrm{~m}, 1 \mathrm{H}, 37-\mathrm{H}), 5.43(\mathrm{~d}, J=$ $10.2 \mathrm{~Hz}, 1 \mathrm{H}, 3-\mathrm{H}), 5.86 \sim 5.89$ (m, 1H, 27-H), 6.61 (d, $J=$ $10.2 \mathrm{~Hz}, 1 \mathrm{H}, 4-\mathrm{H}), 7.37$ (d, J=7.2 Hz, 1H, 10-H); IR (KBr) $v: 3341,2932,2687,2294,2116,1772,1716,1650$, 1588, 1452, 1419, 1381, 1266, 1108, 1075, $754 \mathrm{~cm}^{-1}$; ESI-MS $m / z: 703[\mathrm{M}+\mathrm{H}]^{+}$; HRMS calcd for $\mathrm{C}_{41} \mathrm{H}_{51} \mathrm{O}_{10}$ $(\mathrm{M}+\mathrm{H})^{+}$703.3477, found 703.3465 .

\subsection{7-去异戊烯-17-(1-吗啉基)甲酰基甲基-6-甲氧}

\section{基-藤黄酸甲酯(10)的合成}

将化合物 5 (40 mg, $0.062 \mathrm{mmol}$ )溶于 $5 \mathrm{~mL}$ THF 中, 加入 DMAP $9 \mathrm{mg}(0.074 \mathrm{mmol})$ 和 EDCI $14 \mathrm{mg}(0.074$ $\mathrm{mmol})$ 搅拌 $0.5 \mathrm{~h}$, 冰浴下缓慢加入吗啉 $6 \mu \mathrm{L}(0.069$ $\mathrm{mmol}$ ), 反应 $8 \mathrm{~h}$, 加入饱和 $\mathrm{NH}_{4} \mathrm{Cl}$ 水溶液并搅拌 $5 \mathrm{~min}$, 乙酸乙酯萃取, 分出乙酸乙酯层, 饱和食盐水洗, 无水 硫酸钠干燥, 蒸出溶剂, 柱层析 $[V$ (石油醚) $: V($ 乙酸乙 酯 $)=2: 1$ ] 洗脱, 得到黄色油状物 $16 \mathrm{mg}$, 收率 $36 \% .{ }^{1} \mathrm{H}$ NMR $\left(\mathrm{CDCl}_{3}, 300 \mathrm{MHz}\right) \delta: 1.29(\mathrm{~s}, 3 \mathrm{H}, 19-\mathrm{H}), 1.37 \sim 1.39$ (m, 1H, 21b-H), 1.41 1.44 (m, 1H, 24-H), $1.48(\mathrm{~s}, 3 \mathrm{H}$, 40-H), 1.65 (s, 3H, 39-H), 1.69 (s, 3H, 20-H), 1.71 (s, 3H, 
29-H), 1.74 (s, 3H, 25-H ), $2.08 \sim 2.10$ (m, 2H, 36-H), 2.18 (s, 1H, 21a-H), 2.35 2.37 (m, 4H, 3',5'- $\left.\mathrm{CH}_{2}\right), 2.51$ (d, $J=$ $9.3 \mathrm{~Hz}, 1 \mathrm{H}, 22-\mathrm{H}), 2.97 \sim 3.01(\mathrm{~m}, 2 \mathrm{H}, 26-\mathrm{H}), 3.22 \sim 3.24$ $(\mathrm{m}, 1 \mathrm{H}, 31 \mathrm{a}-\mathrm{H}), 3.31 \sim 3.33(\mathrm{~m}, 1 \mathrm{H}, 31 \mathrm{~b}-\mathrm{H}), 3.42 \sim 3.45$ (m, 1H, 11-H), $3.44\left(\mathrm{~s}, 3 \mathrm{H}, 6-\mathrm{OCH}_{3}\right), 3.57 \sim 3.59(\mathrm{~m}, 4 \mathrm{H}$, $\left.2^{\prime}, 6^{\prime}-\mathrm{CH}_{2}\right), 3.82\left(\mathrm{~s}, 3 \mathrm{H}, \mathrm{COOCH}_{3}\right), 5.03 \sim 5.06(\mathrm{~m}, 1 \mathrm{H}$, $37-\mathrm{H}), 5.52$ (d, $J=10.2 \mathrm{~Hz}, 1 \mathrm{H}, 3-\mathrm{H}), 5.97 \sim 5.99(\mathrm{~m}, 1 \mathrm{H}$, 27-H), 6.69 (d, $J=10.2 \mathrm{~Hz}, 1 \mathrm{H}, 4-\mathrm{H}), 7.44$ (d, $J=6.9 \mathrm{~Hz}$, $1 \mathrm{H}, 10-\mathrm{H})$; IR (KBr) v: 3342, 2964, 2927, 2303, 1721, $1657,1587,1516,1456,1426,1380,1233,1174,1133$, 1105, 1036, $755 \mathrm{~cm}^{-1}$; ESI-MS $716[\mathrm{M}+\mathrm{H}]^{+}$; HRMS calcd for $\mathrm{C}_{41} \mathrm{H}_{49} \mathrm{O}_{10} \mathrm{NNa}(\mathrm{M}+\mathrm{Na})^{+} 738.3249$, found 738.3227.

\subsection{7-去异戊烯-17-(1-哌啶基)甲酰基甲基-6-甲氧 基-藤黄酸甲酯(11)的合成}

11 的合成方法同 10 , 原料为 $\mathbf{5}$ 和哌啶, 得到黄色油 状物 $11 \mathrm{mg}$, 收率 $25 \% .{ }^{1} \mathrm{H} \mathrm{NMR}\left(\mathrm{CDCl}_{3}, 300 \mathrm{MHz}\right) \delta$ : $1.25 \sim 1.27\left(\mathrm{~m}, 2 \mathrm{H}, 4{ }^{\prime}-\mathrm{CH}_{2}\right), 1.29(\mathrm{~s}, 3 \mathrm{H}, 19-\mathrm{H}), 1.37 \sim$ $1.39(\mathrm{~m}, 1 \mathrm{H}, 21 \mathrm{~b}-\mathrm{H}), 1.41 \sim 1.44(\mathrm{~m}, 1 \mathrm{H}, 24-\mathrm{H}), 1.47 \sim$ $1.49\left(\mathrm{~m}, 4 \mathrm{H}, 3^{\prime}, 5^{\prime}-\mathrm{CH}_{2}\right), 1.60(\mathrm{~s}, 3 \mathrm{H}, 40-\mathrm{H}), 1.65(\mathrm{~s}, 3 \mathrm{H}$, 39-H), 1.67 (s, 3H, 20-H), 1.69 (s, 3H, 29-H), 1.74 (s, 3H, 25-H ), $2.07 \sim 2.10$ (m, 2H, 36-H), 2.18 (s, 1H, 21a-H), $2.34 \sim 2.36\left(\mathrm{~m}, 4 \mathrm{H}, 2^{\prime}, 6^{\prime}-\mathrm{H}\right), 2.51(\mathrm{~d}, J=9.3 \mathrm{~Hz}, 1 \mathrm{H}$, $22-\mathrm{H}), 2.97 \sim 2.99(\mathrm{~m}, 2 \mathrm{H}, 26-\mathrm{H}), 3.22 \sim 3.24(\mathrm{~m}, 1 \mathrm{H}$, $31 \mathrm{a}-\mathrm{H}), 3.32 \sim 3.35(\mathrm{~m}, 1 \mathrm{H}, 31 \mathrm{~b}-\mathrm{H}), 3.37 \sim 3.39(\mathrm{~m}, 1 \mathrm{H}$, $11-\mathrm{H}), 3.43\left(\mathrm{~s}, 3 \mathrm{H}, 6-\mathrm{OCH}_{3}\right), 3.82\left(\mathrm{~s}, 3 \mathrm{H}, \mathrm{COOCH}_{3}\right)$, $5.05 \sim 5.07(\mathrm{~m}, 1 \mathrm{H}, 37-\mathrm{H}), 5.54(\mathrm{~d}, J=10.2 \mathrm{~Hz}, 1 \mathrm{H}, 3-\mathrm{H})$, $5.97 \sim 5.99$ (m, 1H, 27-H), 6.69 (d, J=10.2 Hz, 1H, 4-H), 7.44 (d, $J=6.6 \mathrm{~Hz}, 1 \mathrm{H}, 10-\mathrm{H})$; IR (KBr) v: 3406, 2974, 2929, 2363, 1731, 1660, 1585, 1458, 1430, 1379, 1309, 1266, 1225, 1180, 1138, 1038, $754 \mathrm{~cm}^{-1}$; ESI-MS m/z: 714 $[\mathrm{M}+\mathrm{H}]^{+}$; HRMS calcd for $\mathrm{C}_{42} \mathrm{H}_{51} \mathrm{O}_{9} \mathrm{NNa}(\mathrm{M}+\mathrm{Na})^{+}$ 736.3456 , found 736.3437 .

\subsection{7-去异戊烯-17-(1-吡咯烷基)甲酰基甲基-6-甲 氧基-藤黄酸甲酯(12)的合成}

12 的合成方法同 10 , 原料为 $\mathbf{5}$ 和吡咯烷, 得到黄色 油状物 $10 \mathrm{mg}$, 收率 $23 \% .{ }^{1} \mathrm{H}$ NMR $\left(\mathrm{CDCl}_{3}, 300 \mathrm{MHz}\right) \delta$ : $1.29(\mathrm{~s}, 3 \mathrm{H}, 19-\mathrm{H}), 1.37 \sim 1.39(\mathrm{~m}, 1 \mathrm{H}, 21 \mathrm{~b}-\mathrm{H}), 1.42 \sim 1.44$ (m, 1H, 24-H), 1.48 (s, 3H, 40-H), 1.64 (s, 3H, 39-H), 1.69 (s, 3H, 20-H), 1.72 (s, 3H, 29-H), 1.75 (s, 3H, 25-H ), $1.83 \sim 1.85\left(\mathrm{~m}, 4 \mathrm{H}, 3^{\prime}, 4^{\prime}-\mathrm{CH}_{2}\right), 2.09 \sim 2.12(\mathrm{~m}, 2 \mathrm{H}, 36-\mathrm{H})$, $2.18(\mathrm{~s}, 1 \mathrm{H}, 21 \mathrm{a}-\mathrm{H}), 2.26 \sim 2, .68\left(\mathrm{~m}, 4 \mathrm{H}, 2^{\prime}, 5^{\prime}-\mathrm{CH}_{2}\right), 2.51$ (d, $J=9.3 \mathrm{~Hz}, 1 \mathrm{H}, 22-\mathrm{H}), 2.97 \sim 2.98(\mathrm{~m}, 2 \mathrm{H}, 26-\mathrm{H})$, $3.21 \sim 3.24(\mathrm{~m}, 1 \mathrm{H}, 31 \mathrm{a}-\mathrm{H}), 3.26 \sim 3.28(\mathrm{~m}, 1 \mathrm{H}, 31 \mathrm{~b}-\mathrm{H})$, $3.36 \sim 3.38(\mathrm{~m}, 1 \mathrm{H}, 11-\mathrm{H}), 3.43\left(\mathrm{~s}, 3 \mathrm{H}, 6-\mathrm{OCH}_{3}\right), 3.87(\mathrm{~s}$, $\left.3 \mathrm{H}, \mathrm{COOCH}_{3}\right), 5.04 \sim 5.07(\mathrm{~m}, 1 \mathrm{H}, 37-\mathrm{H}), 5.45 \sim 5.47(\mathrm{~m}$, $1 \mathrm{H}, 27-\mathrm{H}), 5.53$ (d, $J=10.2 \mathrm{~Hz}, 1 \mathrm{H}, 3-\mathrm{H}), 6.67$ (d, $J=4.8$ $\mathrm{Hz}, 1 \mathrm{H}, 4-\mathrm{H}), 7.44$ (d, $J=6.9 \mathrm{~Hz}, 1 \mathrm{H}, 10-\mathrm{H})$; IR (KBr) $v$ : 3406, 2973, 2928, 2380, 1731, 1661, 1585, 1458, 1430, 1379, 1309, 1225, 1180, 1138, 1038, $755 \mathrm{~cm}^{-1}$; ESI-MS $m / z: 700[\mathrm{M}+\mathrm{H}]^{+}$; HRMS calcd for $\mathrm{C}_{41} \mathrm{H}_{49} \mathrm{O}_{9} \mathrm{NNa}(\mathrm{M}+$ $\mathrm{Na})^{+} 722.3305$, found 722.3277 .

\subsection{7-去异戊烯-17-(4-甲基-1-哌嗪基)甲酰基甲 基-6-甲氧基-藤黄酸甲酯(13)的合成}

13 的合成方法同 10 , 原料为 $\mathbf{5}$ 和 $N$-甲基哌嗪，得到 黄色油状物 $17 \mathrm{mg}$, 收率 $35 \%$. ${ }^{1} \mathrm{H} \mathrm{NMR}\left(\mathrm{CDCl}_{3}, 300\right.$ $\mathrm{MHz}) \delta: 1.29$ (s, 3H, 19-H), $1.37 \sim 1.39$ (m, 1H, 21b-H), $1.41 \sim 1.44$ (m, 1H, 24-H), 1.48 (s, 3H, 40-H), 1.64 (s, 3H, 39-H), 1.69 (s, 3H, 20-H), 1.70 (s, 3H, 29-H), 1.74 (s, 3H, $25-\mathrm{H}), 2.06 \sim 2.08(\mathrm{~m}, 2 \mathrm{H}, 36-\mathrm{H}), 2.18(\mathrm{~s}, 1 \mathrm{H}, 21 \mathrm{a}-\mathrm{H})$, $2.32\left(\mathrm{~s}, 3 \mathrm{H}, 4^{\prime}-\mathrm{CH}_{3}\right), 2.34 \sim 2.37\left(\mathrm{~m}, 8 \mathrm{H}, 2^{\prime}, 3^{\prime}, 5^{\prime}, 6^{\prime}-\mathrm{CH}_{2}\right)$, 2.52 (d, $J=9.3 \mathrm{~Hz}, 1 \mathrm{H}, 22-\mathrm{H}), 2.96 \sim 2.98$ (m, 2H, 26-H), $3.25 \sim 3.27(\mathrm{~m}, 1 \mathrm{H}, 31 \mathrm{a}-\mathrm{H}), 3.33 \sim 3.35(\mathrm{~m}, 1 \mathrm{H}, 31 \mathrm{~b}-\mathrm{H})$, $3.38 \sim 3.40(\mathrm{~m}, 1 \mathrm{H}, 11-\mathrm{H}), 3.43\left(\mathrm{~s}, 3 \mathrm{H}, 6-\mathrm{OCH}_{3}\right), 3.83(\mathrm{~s}$, $\left.3 \mathrm{H}, \mathrm{COOCH}_{3}\right), 5.03 \sim 5.05(\mathrm{~m}, 1 \mathrm{H}, 37-\mathrm{H}), 5.52(\mathrm{~d}, J=2.1$ $\mathrm{Hz}, 1 \mathrm{H}, 3-\mathrm{H}), 5.57 \sim 5.99(\mathrm{~m}, 1 \mathrm{H}, 27-\mathrm{H}), 6.70$ (d, $J=10.2$ $\mathrm{Hz}, 1 \mathrm{H}, 4-\mathrm{H}), 7.44$ (d, $J=6.9 \mathrm{~Hz}, 1 \mathrm{H}, 10-\mathrm{H})$; IR (KBr) v: 3405, 2968, 2926, 2362, 1726, 1660, 1586, 1455, 1425, 1381, 1265, 1223, 1144, 1097, 1036, $755 \mathrm{~cm}^{-1}$; ESI-MS $m / z: 729[\mathrm{M}+\mathrm{H}]^{+}$; HRMS calcd for $\mathrm{C}_{42} \mathrm{H}_{53} \mathrm{O}_{9} \mathrm{~N}_{2}(\mathrm{M}+$ $\mathrm{H})^{+} 729.3746$, found 729.3728 .

\subsection{7-去异戊烯-17-( $N, N$-二乙基胺基)甲酰基甲基- 6-甲氧基-藤黄酸甲酯(14)的合成}

14 的合成方法同 10 , 原料为 5 和 $N, N$-二乙胺, 得到 黄色油状物 $10 \mathrm{mg}$, 收率 $23 \% .{ }^{1} \mathrm{H}$ NMR $\left(\mathrm{CDCl}_{3}, 300\right.$ $\mathrm{MHz}) \delta: 1.17 \sim 1.19\left(\mathrm{~m}, 6 \mathrm{H}, 3^{\prime}, 3^{\prime \prime}-\mathrm{CH}_{3}\right), 1.29(\mathrm{~s}, 3 \mathrm{H}$, $19-\mathrm{H}), 1.38 \sim 1.40(\mathrm{~m}, 1 \mathrm{H}, 21 \mathrm{~b}-\mathrm{H}), 1.41 \sim 1.44(\mathrm{~m}, 1 \mathrm{H}$, 24-H), 1.48 (s, 3H, 40-H), 1.64 (s, 3H, 39-H), 1.69 (s, 3H, 20-H), 1.71 (s, 3H, 29-H), 1.75 (s, 3H, 25-H ), 2.12 (m, $2 \mathrm{H}, 36-\mathrm{H}), 2.18(\mathrm{~s}, 1 \mathrm{H}, 21 \mathrm{a}-\mathrm{H}), 2.33 \sim 2.35(\mathrm{~m}, 4 \mathrm{H}$, 2', 2"- $\left.\mathrm{CH}_{2}\right), 2.51(\mathrm{~d}, J=9.3 \mathrm{~Hz}, 1 \mathrm{H}, 22-\mathrm{H}), 2.97 \sim 2.99(\mathrm{~m}$, $2 \mathrm{H}, 26-\mathrm{H}), 3.21 \sim 3.24(\mathrm{~m}, 1 \mathrm{H}, 31 \mathrm{a}-\mathrm{H}), 3.31 \sim 3.34(\mathrm{~m}$, $1 \mathrm{H}, 31 \mathrm{~b}-\mathrm{H}), 3.36 \sim 3.28(\mathrm{~m}, 1 \mathrm{H}, 11-\mathrm{H}), 3.43(\mathrm{~s}, 3 \mathrm{H}$, $\left.6-\mathrm{OCH}_{3}\right), 3.81\left(\mathrm{~s}, 3 \mathrm{H}, \mathrm{COOCH}_{3}\right), 5.04 \sim 5.06(\mathrm{~m}, 1 \mathrm{H}$, $37-\mathrm{H}), 5.54$ (d, $J=10.2 \mathrm{~Hz}, 1 \mathrm{H}, 3-\mathrm{H}), 5.97 \sim 5.99(\mathrm{~m}, 1 \mathrm{H}$, 27-H), 6.68 (d, $J=10.2 \mathrm{~Hz}, 1 \mathrm{H}, 4-\mathrm{H}), 7.44$ (d, $J=6.9 \mathrm{~Hz}$, $1 \mathrm{H}, 10-\mathrm{H})$; IR (KBr) v: 3404, 3342, 2964, 2928, 2371, 1726, 1658, 1587, 1455, 1431, 1381, 1265, 1145, 1106, $754 \mathrm{~cm}^{-1}$; ESI-MS m/z: $702[\mathrm{M}+\mathrm{H}]^{+}$; HRMS calcd for $\mathrm{C}_{41} \mathrm{H}_{51} \mathrm{O}_{9} \mathrm{NNa}(\mathrm{M}+\mathrm{Na})^{+}$724.3456, found 724.3433 . 
1.14 17-去异戊烯-17-(4-甲基苯胺基)甲酰基甲基-6甲氧基-藤黄酸甲酯(15)的合成

15 的合成方法同 10 , 原料为 5 和 4-甲基苯胺, 得到 黄色油状物 $16 \mathrm{mg}$, 收率 $35 \% .{ }^{1} \mathrm{H}$ NMR $\left(\mathrm{CDCl}_{3}, 300\right.$ MHz) $\delta: 1.26$ (s, 3H, 4'-CH CH $_{3}, 1.29$ (s, 3H, 19-H), 1.39 1.42 (m, 2H, 21b-H, 24-H), 1.51 (s, 3H, 40-H), 1.66 (s, 3H, 39-H), 1.66 (s, 3H, 20-H), 1.68 (s, 3H, 29-H), 1.78 (s, 3H, $25-\mathrm{H}), 2.07 \sim 2.09(\mathrm{~m}, 2 \mathrm{H}, 36-\mathrm{H}), 2.18(\mathrm{~s}, 1 \mathrm{H}, 21 \mathrm{a}-\mathrm{H})$, $2.48(\mathrm{~d}, J=9.3 \mathrm{~Hz}, 1 \mathrm{H}, 22-\mathrm{H}), 2.96 \sim 2.98(\mathrm{~m}, 2 \mathrm{H}, 26-\mathrm{H})$, $3.24 \sim 3.26(\mathrm{~m}, 1 \mathrm{H}, 31 \mathrm{a}-\mathrm{H}), 3.34 \sim 3.37(\mathrm{~m}, 1 \mathrm{H}, 31 \mathrm{~b}-\mathrm{H})$, $3.38(\mathrm{~s}, 1 \mathrm{H}, 11-\mathrm{H}), 3.43\left(\mathrm{~s}, 3 \mathrm{H}, 6-\mathrm{OCH}_{3}\right), 3.84(\mathrm{~s}, 3 \mathrm{H}$, $\left.\mathrm{COOCH}_{3}\right), 5.08 \sim 5.11(\mathrm{~m}, 1 \mathrm{H}, 37-\mathrm{H}), 5.53(\mathrm{~d}, J=10.2$ $\mathrm{Hz}, 1 \mathrm{H}, 3-\mathrm{H}), 5.94 \sim 5.96(\mathrm{~m}, 1 \mathrm{H}, 27-\mathrm{H}), 6.70$ (d, $J=10.2$ Hz, 1H, 4-H), 6.96 (s, 1H, NH), 7.09 (d, $J=8.4 \mathrm{~Hz}, 2 \mathrm{H}$, 3',5'-H), 7.30 (d, $\left.J=8.4 \mathrm{~Hz}, 2 \mathrm{H}, 2^{\prime}, 6^{\prime}-\mathrm{H}\right), 7.44$ (d, $J=6.9$ $\mathrm{Hz}, 1 \mathrm{H}, 10-\mathrm{H})$; IR (KBr) v: 3325, 2965, 2925, 2860, 2535, 2393, 2225, 2079, 1970, 1894, 1725, 1659, 1588, 1524, $1455,1431,1378,1311,1229,1179,1135,1039,754$ $\mathrm{cm}^{-1}$; ESI-MS m/z: $736[\mathrm{M}+\mathrm{H}]^{+}$; HRMS calcd for $\mathrm{C}_{44} \mathrm{H}_{50} \mathrm{O}_{9} \mathrm{~N}(\mathrm{M}+\mathrm{H})^{+}$736.3486, found 736.3474 .

1.15 17-去异戊烯-17-(4-甲氧基苯胺基)甲酰基甲基6-甲氧基-藤黄酸甲酯(16)的合成

16 的合成方法同 10 , 原料为 5 和 4-甲氧基苯胺, 得 到黄色粘稠状物 $15 \mathrm{mg}$, 收率 $32 \% .{ }^{1} \mathrm{H}$ NMR $\left(\mathrm{CDCl}_{3}, 300\right.$ MHz) $\delta: 1.29$ (s, 3H, 19-H), 1.37 1.40 (m, 1H, 21b-H), $1.44 \sim 1.47$ (m, 1H, 24-H), 1.51 (s, 3H, 40-H), 1.62 (s, 3H, 39-H), 1.67 (s, 3H, 20-H), 1.68 (s, 3H, 29-H), 1.78 (s, 3H, $25-\mathrm{H}), 2.08 \sim 2.10(\mathrm{~m}, 2 \mathrm{H}, 36-\mathrm{H}), 2.18(\mathrm{~s}, 1 \mathrm{H}, 21 \mathrm{a}-\mathrm{H})$, 2.49 (d, $J=9.3 \mathrm{~Hz}, 1 \mathrm{H}, 22-\mathrm{H}), 2.96 \sim 2.98(\mathrm{~m}, 2 \mathrm{H}, 26-\mathrm{H})$, $3.12(\mathrm{~m}, 1 \mathrm{H}, 31 \mathrm{a}-\mathrm{H}), 3.25 \sim 3.27(\mathrm{~m}, 1 \mathrm{H}, 31 \mathrm{~b}-\mathrm{H}), 3.34(\mathrm{~s}$, $1 \mathrm{H}, 11-\mathrm{H}), 3.43$ (s, 3H, 6- $\left.\mathrm{OCH}_{3}\right), 3.77$ (s, 3H, 4'-- $\left.\mathrm{OCH}_{3}\right)$, $3.81\left(\mathrm{~s}, 3 \mathrm{H}, \mathrm{COOCH}_{3}\right), 5.07 \sim 5.09(\mathrm{~m}, 1 \mathrm{H}, 37-\mathrm{H}), 5.54(\mathrm{~d}$, $J=10.2 \mathrm{~Hz}, 1 \mathrm{H}, 3-\mathrm{H}), 5.94 \sim 5.97(\mathrm{~m}, 1 \mathrm{H}, 27-\mathrm{H}), 6.71(\mathrm{~d}$, $J=10.2 \mathrm{~Hz}, 1 \mathrm{H}, 4-\mathrm{H}), 6.82\left(\mathrm{~d}, J=9.0 \mathrm{~Hz}, 2 \mathrm{H}, 3^{\prime}, 5^{\prime}-\mathrm{H}\right)$, 6.92 (s, 1H, NH), 7.32 (d, $\left.J=9.0 \mathrm{~Hz}, 2 \mathrm{H}, 2^{\prime}, 6^{\prime}-\mathrm{H}\right), 7.44$ (d, $J=6.9 \mathrm{~Hz}, 1 \mathrm{H}, 10-\mathrm{H}$ ); IR (KBr) $v: 3324,2965,2925$, 2859, 2528, 2364, 2227, 2089, 1725, 1658, 1588, 1524, $1455,1431,1378,1311,1229,1179,1135,1039,755$ $\mathrm{cm}^{-1}$; ESI-MS m/z: $752[\mathrm{M}+\mathrm{H}]^{+}$; HRMS calcd for $\mathrm{C}_{44} \mathrm{H}_{50} \mathrm{O}_{10} \mathrm{~N}(\mathrm{M}+\mathrm{H})^{+}$752.3429, found 752.3424.

1.16 17-去异戊烯-17-(4-氯苯胺基)甲酰基甲基-6-甲 氧基-藤黄酸甲酯(17)的合成

17 的合成方法同 10 , 原料为 5 和 4-氯苯胺, 得到黄 色粘稠状物 $20 \mathrm{mg}$, 收率 $43 \% .{ }^{1} \mathrm{H}$ NMR $\left(\mathrm{CDCl}_{3}, 300\right.$
MHz) $\delta: 1.26$ (s, 3H, 19-H), $1.28 \sim 1.29$ (m, 3H, 24-H), $1.38 \sim 1.40(\mathrm{~m}, 1 \mathrm{H}, 21 \mathrm{~b}-\mathrm{H}), 1.51(\mathrm{~s}, 3 \mathrm{H}, 40-\mathrm{H}), 1.65(\mathrm{~s}$, 3H, 39-H), 1.66 (s, 2H, 20-H), 1.68 (s, 3H, 29-H), 1.77 (s, $3 \mathrm{H}, 25-\mathrm{H}), 2.08 \sim 2.11(\mathrm{~m}, 2 \mathrm{H}, 36-\mathrm{H}), 2.17(\mathrm{~s}, 1 \mathrm{H}, 21 \mathrm{a}-\mathrm{H})$, $2.46(\mathrm{~d}, J=9.0 \mathrm{~Hz}, 1 \mathrm{H}, 22-\mathrm{H}), 2.94 \sim 2.96(\mathrm{~m}, 2 \mathrm{H}, 26-\mathrm{H})$, $3.23 \sim 3.25(\mathrm{~m}, 1 \mathrm{H}, 31 \mathrm{a}-\mathrm{H}), 3.27 \sim 3.30(\mathrm{~m}, 1 \mathrm{H}, 31 \mathrm{~b}-\mathrm{H})$, $3.42\left(\mathrm{~s}, 3 \mathrm{H}, 6-\mathrm{OCH}_{3}\right), 3.44(\mathrm{~s}, 1 \mathrm{H}, 11-\mathrm{H}), 3.81(\mathrm{~s}, 3 \mathrm{H}$, $\left.\mathrm{COOCH}_{3}\right), 5.07 \sim 5.09(\mathrm{~m}, 1 \mathrm{H}, 37-\mathrm{H}), 5.52(\mathrm{~d}, J=10.2$ $\mathrm{Hz}, 1 \mathrm{H}, 3-\mathrm{H}), 5.94 \sim 5.97$ (m, 1H, 27-H), 6.72 (d, $J=10.5$ Hz, 1H, 4-H), 7.05 (s, 1H, NH), 7.22 (d, $J=8.7 \mathrm{~Hz}, 2 \mathrm{H}$, 3',5'-H), 7.36 (d, $\left.J=8.7 \mathrm{~Hz}, 2 \mathrm{H}, 2^{\prime}, 6^{\prime}-\mathrm{H}\right), 7.43$ (d, $J=6.9$ $\mathrm{Hz}, 1 \mathrm{H}, 10-\mathrm{H}) ;{ }^{13} \mathrm{C}$ NMR $\left(\mathrm{CDCl}_{3}, 75 \mathrm{MHz}\right) \delta: 17.7(\mathrm{C}-40)$, 20.3 (C-29), 21.6 (C-36), 24.9 (C-21), 25.2 (C-39), 28.8 (C-19), 28.9 (C-24), 29.4 (C-26), 30.4 (C-25), 31.9 (C-31), 36.9 (C-20), 46.3 (C-11), 48.5 (C-22), 50.4 (30-COOMe), 61.7 (6-OMe), 79.8 (C-2), 83.0 (C-13), 83.2 (C-23), 90.5 (C-14), 107.0 (C-7), 108.6 (C-5), 112.1 (C-17), 117.0 (C-4), 120.2 (Ph-C), 121.2 (C-37), 125.6 (Ph-C), 127.0 (C-3), 128.4 (C-28), 131.6 (Ph-C), 133.5 (C-38), 135.2 (C-9), 135.8 (C-10), 135.9 (C-27), 154.9 (C-16), 157.7 (C-6), 159.4 (C-18), 166.8 (C-32), 169.9 (C-30), 174.2 (C-8), 203.3 (C-12); IR (KBr) v: 3325, 3194, 2970, 2928, 2246, 1777, 1707, 1658, 1588, 1530, 1456, 1431, 1383, 1312, 1230, 1178, 1136, 1102, 1046, $726 \mathrm{~cm}^{-1}$; ESI-MS $m / z: 756[\mathrm{M}+\mathrm{H}]^{+}$; HRMS calcd for $\mathrm{C}_{43} \mathrm{H}_{47} \mathrm{O}_{9} \mathrm{NCl}(\mathrm{M}+$ H) ${ }^{+} 756.2934$, found 756.2928 .

1.17 17-去异戊烯-17-(3-硝基-苯胺基)甲酰基甲基-6甲氧基-藤黄酸甲酯(18)的合成

18 的合成方法同 10 , 原料为 5 和 3-硝基苯胺, 得到 黄色粘稠状物 $15 \mathrm{mg}$, 收率 $32 \% .{ }^{1} \mathrm{H}$ NMR $\left(\mathrm{CDCl}_{3}, 300\right.$ MHz) $\delta: 1.26(\mathrm{~s}, 3 \mathrm{H}, 19-\mathrm{H}), 1.27 \sim 1.29(\mathrm{~m}, 1 \mathrm{H}, 24-\mathrm{H})$, $1.42 \sim 1.44(\mathrm{~m}, 1 \mathrm{H}, 21 \mathrm{~b}-\mathrm{H}), 1.52$ (s, 3H, 40-H), 1.64 (s, $3 \mathrm{H}, 39-\mathrm{H}), 1.66$ (s, 2H, 20-H), 1.69 (s, 3H, 29-H), 1.78 (s, $3 \mathrm{H}, 25-\mathrm{H}), 2.08 \sim 2.10(\mathrm{~m}, 2 \mathrm{H}, 36-\mathrm{H}), 2.17(\mathrm{~s}, 1 \mathrm{H}, 21 \mathrm{a}-\mathrm{H})$, $2.46 \sim 2.48(\mathrm{~m}, 1 \mathrm{H}, 22-\mathrm{H}), 2.95 \sim 2.97(\mathrm{~m}, 2 \mathrm{H}, 26-\mathrm{H})$, $3.23 \sim 3.25(\mathrm{~m}, 1 \mathrm{H}, 31 \mathrm{a}-\mathrm{H}), 3.29 \sim 3.31(\mathrm{~m}, 1 \mathrm{H}, 31 \mathrm{~b}-\mathrm{H})$, 3.41(s, 1H, 11-H), $3.43\left(\mathrm{~s}, 3 \mathrm{H}, 6-\mathrm{OCH}_{3}\right), 3.78(\mathrm{~s}, 3 \mathrm{H}$, $\left.\mathrm{COOCH}_{3}\right), 5.08 \sim 5.10(\mathrm{~m}, 1 \mathrm{H}, 37-\mathrm{H}), 5.53(\mathrm{~d}, J=10.2$ $\mathrm{Hz}, 1 \mathrm{H}, 3-\mathrm{H}), 5.95 \sim 5.97(\mathrm{~m}, 1 \mathrm{H}, 27-\mathrm{H}), 6.71(\mathrm{~d}, J=10.2$ $\mathrm{Hz}, 1 \mathrm{H}, 4-\mathrm{H}), 7.43 \sim 7.45(\mathrm{~m}, 1 \mathrm{H}, 10-\mathrm{H}), 7.46 \sim 7.48(\mathrm{~m}$, $\left.2 \mathrm{H}, 4^{\prime}, 5^{\prime}-\mathrm{H}\right), 7.91$ (d, $\left.J=8.1 \mathrm{~Hz}, 2 \mathrm{H}, 2^{\prime}, 6^{\prime}-\mathrm{H}\right), 8.18$ (s, 1H, $\mathrm{NH})$; IR (KBr) v: 3325, 3127, 3084, 2968, 2928, 2364, 2248, 1707, 1656, 1588, 1533, 1457, 1429, 1378, 1353, 1314, 1229, 1178, 1135, 1106, 1043, $729 \mathrm{~cm}^{-1}$; ESI-MS m/z: $767[\mathrm{M}+\mathrm{H}]^{+}$; HRMS calcd for $\mathrm{C}_{43} \mathrm{H}_{47} \mathrm{O}_{11} \mathrm{~N}_{2}$ (M+ 
H) ${ }^{+}$767.3174, found 767.3177 .

\subsection{7-去异戊烯-17-(丁二酰亚胺氧基甲酰基)甲 基-6-甲氧基-藤黄酸甲酯(6)的合成}

将化合物 5 (40 mg, $0.062 \mathrm{mmol}$ )溶于 $5 \mathrm{~mL}$ 乙醇中, 加入 DMAP $9 \mathrm{mg}(0.074 \mathrm{mmol})$ 和 DCC $15 \mathrm{mg}(0.074$ $\mathrm{mmol}$ ), 摚拌 $0.5 \mathrm{~h}$ 后, 加入 $N$-差基琥珀酰亚胺 $9 \mathrm{mg}$ $(0.078 \mathrm{mmol})$, 室温搅拌 $3 \mathrm{~h}$, 再加入饱和 $\mathrm{NH}_{4} \mathrm{Cl}$ 水溶液 并摚拌 $5 \mathrm{~min}$, 用乙酸乙酯萃取, 分出乙酸乙酯层, 用饱 和食盐水洗, 无水硫酸钠干燥, 蒸出溶剂, 得到黄色油 状物 $20 \mathrm{mg}$, 收率 43\%. ESI-MS m/z: $744[\mathrm{M}+\mathrm{H}]^{+}$.

\subsection{7-去异戊烯-17-( $N$-羧基甲基)胺基甲酰基甲} 基-6-甲氧基-藤黄酸甲酯(19)的合成

将化合物 6 (40 mg, $0.054 \mathrm{mmol}$ ) 溶于 $2 \mathrm{~mL}$ DMSO 中, 加入甘氨酸 $4 \mathrm{mg}(0.054 \mathrm{mmol})$ 和三乙胺 $0.4 \mathrm{~mL}$, 摚 拌反应 $8 \mathrm{~h}$, 再加入饱和 $\mathrm{NH}_{4} \mathrm{Cl}$ 水溶液并摚拌, 乙酸乙 酯萃取, 分出乙酸乙酯层, 用饱和食盐水洗, 无水硫酸 钠干燥, 蒸出溶剂, 柱层析 $[V$ ( 乙酸乙酯) $: V($ 甲醇 $)=$ $16 ： 1]$ 洗脱, 得黄色油状物 $11 \mathrm{mg}$, 收率 $29 \% .{ }^{1} \mathrm{H}$ NMR $\left(\mathrm{CDCl}_{3}, 300 \mathrm{MHz}\right) \delta: 1.27(\mathrm{~s}, 3 \mathrm{H}, 19-\mathrm{H}), 1.29 \sim 1.31(\mathrm{~m}$, $3 \mathrm{H}, 24-\mathrm{H}), 1.37 \sim 1.39$ (m, 1H, 21b-H), 1.47 (s, 3H, 40-H), 1.66 (s, 3H, 39-H), 1.68 (s, 2H, 20-H), 1.70 (s, 3H, 29-H), 1.76 (s, 3H, 25-H), 2.05 2.07 (m, 2H, 36-H), 2.29 (s, 1H, $21 \mathrm{a}-\mathrm{H}), 2.55(\mathrm{~d}, J=9.3 \mathrm{~Hz}, 1 \mathrm{H}, 22-\mathrm{H}), 2.95 \sim 2.98(\mathrm{~m}, 2 \mathrm{H}$, $26-\mathrm{H}), 3.06 \sim 3.08\left(\mathrm{~m}, 2 \mathrm{H}, \mathrm{NHCH}_{2} \mathrm{COOH}\right), 3.14 \sim 3.16$ (m, 1H, 31a-H), 3.28 3.30 (m, 1H, 31b-H), $3.42(\mathrm{~s}, 3 \mathrm{H}$, $\left.6-\mathrm{OCH}_{3}\right), 3.60(\mathrm{~s}, 1 \mathrm{H}, 11-\mathrm{H}), 3.81\left(\mathrm{~s}, 3 \mathrm{H}, \mathrm{COOCH}_{3}\right)$, $5.07 \sim 5.09(\mathrm{~m}, 1 \mathrm{H}, 37-\mathrm{H}), 5.53(\mathrm{~d}, J=10.2 \mathrm{~Hz}, 1 \mathrm{H}, 3-\mathrm{H})$, $5.92 \sim 5.94(\mathrm{~m}, 1 \mathrm{H}, 27-\mathrm{H}), 6.45(\mathrm{~s}, 1 \mathrm{H}, \mathrm{NH}), 6.66(\mathrm{~d}, J=$ $10.2 \mathrm{~Hz}, 1 \mathrm{H}, 4-\mathrm{H}), 7.44$ (d, $J=6.9 \mathrm{~Hz}, 1 \mathrm{H}, 10-\mathrm{H})$; IR (KBr) v: 3325, 3191, 2972, 2928, 2364, 2245, 2089, 1893, $1722,1707,1658,1588,1530,1456,1383,1312,1228$, 1177, 1135, 1097, 1042, $752 \mathrm{~cm}^{-1}$; ESI-MS m/z: 704 [M+ $\mathrm{H}]^{+}$; HRMS calcd for $\mathrm{C}_{39} \mathrm{H}_{46} \mathrm{O}_{11} \mathrm{~N}(\mathrm{M}+\mathrm{H})^{+}$: 704.3065, found 704.3053 .

\subsection{7-去异戊烯-17-[N-(1-羧基-2-甲基)丙基]胺基甲 酰基甲基-6-甲氧基-藤黄酸甲酯(20)的合成}

20 的合成方法同 19 , 原料为 $\mathbf{6}$ 和倾氨酸, 得黄色粘 稠物 $13 \mathrm{mg}$, 收率 $33 \% .{ }^{1} \mathrm{H}$ NMR $\left(\mathrm{CDCl}_{3}, 300 \mathrm{MHz}\right) \delta$ : $0.85 \sim 0.86\left[\mathrm{~m}, 7 \mathrm{H}, \mathrm{CH}\left(\mathrm{CH}_{3}\right)_{2}\right], 1.26(\mathrm{~s}, 3 \mathrm{H}, 19-\mathrm{H}), 1.27 \sim$ 1.29 (m, 3H, 24-H), 1.38 1.39 (m, 1H, 21b-H), 1.45 (s, $3 \mathrm{H}, 40-\mathrm{H}), 1.66$ (s, 3H, 39-H), 1.69 (s, 2H, 20-H), 1.75 (s, $3 \mathrm{H}, 29-\mathrm{H}), 1.79$ (s, 3H, 25-H), 2.01 2.03 (m, 2H, 36-H), $2.18(\mathrm{~s}, 1 \mathrm{H}, 21 \mathrm{a}-\mathrm{H}), 2.53(\mathrm{~d}, J=9.0 \mathrm{~Hz}, 1 \mathrm{H}, 22-\mathrm{H}), 2.94 \sim$ $2.96(\mathrm{~m}, 2 \mathrm{H}, 26-\mathrm{H}), 3.19 \sim 3.21(\mathrm{~m}, 1 \mathrm{H}, 31 \mathrm{a}-\mathrm{H}), 3.26 \sim$ $3.28(\mathrm{~m}, 1 \mathrm{H}, 31 \mathrm{~b}-\mathrm{H}), 3.42\left(\mathrm{~s}, 3 \mathrm{H}, 6-\mathrm{OCH}_{3}\right), 3.59(\mathrm{~s}, 1 \mathrm{H}$, $11-\mathrm{H}), 3.78\left(\mathrm{~s}, 3 \mathrm{H}, \mathrm{COOCH}_{3}\right), 4.11(\mathrm{~s}, 1 \mathrm{H}, \mathrm{CH}), 5.09 \sim$ $5.11(\mathrm{~m}, 1 \mathrm{H}, 37-\mathrm{H}), 5.52(\mathrm{~d}, J=10.2 \mathrm{~Hz}, 1 \mathrm{H}, 3-\mathrm{H}), 5.95 \sim$ $5.97(\mathrm{~m}, 1 \mathrm{H}, 27-\mathrm{H}), 6.32(\mathrm{~s}, 1 \mathrm{H}, \mathrm{NH}), 6.65(\mathrm{~d}, J=10.2 \mathrm{~Hz}$, $1 \mathrm{H}, 4-\mathrm{H}), 7.42$ (d, $J=6.6 \mathrm{~Hz}, 1 \mathrm{H}, 10-\mathrm{H})$; IR (KBr) $v: 3325$, 3127, 3084, 2970, 2928, 2364, 2246, 1708, 1656, 1588, $1532,1457,1430,1378,1353,1314,1227,1178,1135$, 1106, 1042, $750 \mathrm{~cm}^{-1}$; ESI-MS m/z: $746[\mathrm{M}+\mathrm{H}]^{+}$; HRMS calcd for $\mathrm{C}_{42} \mathrm{H}_{52} \mathrm{O}_{11} \mathrm{~N}(\mathrm{M}+\mathrm{H})^{+}$: 746.3535, found 746.3532 .

1.21 17-去异戊烯-17-[N-(1-羧基-3-甲硫基)丙基]胺基 甲酰基甲基-6-甲氧基-藤黄酸甲酯(21)的合成

21 的合成方法同 19 , 原料为 6 和甲硫氨酸, 得黄色 粘稠物 $10 \mathrm{mg}$, 收率 $24 \% .{ }^{1} \mathrm{H}$ NMR $\left(\mathrm{CDCl}_{3}, 300 \mathrm{MHz}\right) \delta$ : 1.26 (s, 3H, 19-H), $1.27 \sim 1.28$ (m, 3H, 24-H), $1.37 \sim 1.39$ (m, 1H, 21b-H), 1.45 (s, 3H, 40-H), $1.65(\mathrm{~s}, 3 \mathrm{H}, 39-\mathrm{H})$, 1.69 (s, 2H, 20-H), 1.75 (s, 3H, 29-H), 1.80 (s, 3H, 25-H), $1.93 \sim 1.95\left(\mathrm{~m}, 7 \mathrm{H}, \mathrm{CH}_{2} \mathrm{CH}_{2} \mathrm{SCH}_{3}\right), 2.02 \sim 2.03(\mathrm{~m}, 2 \mathrm{H}$, 36-H), 2.18 (s, 1H, 21a-H), 2.54 (d, $J=9.3 \mathrm{~Hz}, 1 \mathrm{H}, 22-\mathrm{H})$, $2.95 \sim 2.97(\mathrm{~m}, 2 \mathrm{H}, 26-\mathrm{H}), 3.22 \sim 3.24(\mathrm{~m}, 1 \mathrm{H}, 31 \mathrm{a}-\mathrm{H})$, $3.30 \sim 3.32(\mathrm{~m}, 1 \mathrm{H}, 31 \mathrm{~b}-\mathrm{H}), 3.42\left(\mathrm{~s}, 3 \mathrm{H}, 6-\mathrm{OCH}_{3}\right), 3.59$ (s, $1 \mathrm{H}, 11-\mathrm{H}), 3.78\left(\mathrm{~s}, 3 \mathrm{H}, \mathrm{COOCH}_{3}\right), 4.21(\mathrm{~s}, 1 \mathrm{H}, \mathrm{CH})$, $5.10 \sim 5.12(\mathrm{~m}, 1 \mathrm{H}, 37-\mathrm{H}), 5.53(\mathrm{~d}, J=10.2 \mathrm{~Hz}, 1 \mathrm{H}, 3-\mathrm{H})$, $5.96 \sim 5.97(\mathrm{~m}, 1 \mathrm{H}, 27-\mathrm{H}), 6.65(\mathrm{~d}, J=10.2 \mathrm{~Hz}, 1 \mathrm{H}, 4-\mathrm{H})$, 6.87 (s, 1H, NH), 7.42 (d, $J=6.9 \mathrm{~Hz}, 1 \mathrm{H}, 10-\mathrm{H})$; IR (KBr) $v: 3402,2971,2933,2744,2674,2490,1716,1654,1587$, $1458,1424,1307,1230,1180,1136,1037,755 \mathrm{~cm}^{-1}$; ESI-MS $m / z$ : $778[\mathrm{M}+\mathrm{H}]^{+}$; HRMS calcd for $\mathrm{C}_{42} \mathrm{H}_{52} \mathrm{O}_{11} \mathrm{NS}$ $(\mathrm{M}+\mathrm{H})^{+}$778.3256, found 778.3246.

\subsection{7-去异戊烯-17-[ $N$-(1-羧基-2-颈基)乙基]胺基甲} 酰基甲基-6-甲氧基-藤黄酸甲酯(22)的合成

22 的合成方法同 19 , 原料为 6 和半胱氨酸, 得黄色 粘稠物 $9 \mathrm{mg}$, 收率 $23 \% .{ }^{1} \mathrm{H}$ NMR $\left(\mathrm{CDCl}_{3}, 300 \mathrm{MHz}\right) \delta$ : 1.26 (s, 3H, 19-H), $1.27 \sim 1.29$ (m, 3H, 24-H), $1.38 \sim 1.39$ (m, 1H, 21b-H), 1.45 (s, 3H, 40-H), 1.65 (s, 3H, 39-H), 1.69 (s, 2H, 20-H), 1.75 (s, 3H, 29-H), 1.79 (s, 3H, 25-H), $2.02 \sim 2.04(\mathrm{~m}, 2 \mathrm{H}, 36-\mathrm{H}), 2.18(\mathrm{~s}, 1 \mathrm{H}, 21 \mathrm{a}-\mathrm{H}), 2.52(\mathrm{~d}$, $J=9.3 \mathrm{~Hz}, 1 \mathrm{H}, 22-\mathrm{H}), 2.94 \sim 2.95$ (m, 2H, 26-H), 3.20 $3.22(\mathrm{~m}, 1 \mathrm{H}, 31 \mathrm{a}-\mathrm{H}), 3.26 \sim 3.27(\mathrm{~m}, 1 \mathrm{H}, 31 \mathrm{~b}-\mathrm{H}), 3.42(\mathrm{~s}$, $\left.3 \mathrm{H}, 6-\mathrm{OCH}_{3}\right), 3.59(\mathrm{~s}, 1 \mathrm{H}, 11-\mathrm{H}), 3.78\left(\mathrm{~s}, 3 \mathrm{H}, \mathrm{COOCH}_{3}\right)$, $4.19(\mathrm{~s}, 1 \mathrm{H}, \mathrm{CH}), 4.92(\mathrm{~s}, 1 \mathrm{H}, \mathrm{NH}), 5.06 \sim 5.07(\mathrm{~m}, 1 \mathrm{H}$, $37-\mathrm{H}), 5.50(\mathrm{~d}, J=10.2 \mathrm{~Hz}, 1 \mathrm{H}, 3-\mathrm{H}), 5.95 \sim 5.96(\mathrm{~m}, 1 \mathrm{H}$, 27-H), 6.64 (d, $J=10.2 \mathrm{~Hz}, 1 \mathrm{H}, 4-\mathrm{H}), 7.42$ (d, $J=6.9 \mathrm{~Hz}$, 1H, 10-H); IR (KBr) v: 3384, 2970, 2933, 2799, 2744, 2674, 2529, 2489, 1717, 1654, 1587, 1457, 1424, 1360, 
1229, 1178, 1135, 1038, $755 \mathrm{~cm}^{-1}$; ESI-MS m/z: $750[\mathrm{M}+$ $\mathrm{H}]^{+}$; HRMS calcd for $\mathrm{C}_{40} \mathrm{H}_{48} \mathrm{NO}_{11} \mathrm{~S}(\mathrm{M}+\mathrm{H})^{+} 750.2870$, found 750.2891 .

\subsection{3 化合物的生物活性测试}

采用 MTT 法对合成的 17 个藤黄酸衍生物 5, 7 22 进行体外抗肿瘤细胞 HepG2(人肝癌细胞) 、HCT-116(人 结肠癌细胞)的生物活性笁选, 以藤黄酸作为阳性对照.

实验方法: 取对数生长期细胞培养于 96 孔培养板 内, 每孔 $100 \mu \mathrm{L}$ (含 $1000 \sim 2000$ 个肿瘤细胞). 次日, 给 药组加入含有不同浓度化合物, 每药设 4 5 个剂量组, 每组至少设 3 个平行孔. 对照组加入与化合物等体积的 溶剂. 置于 $5 \% \mathrm{CO}_{2}$ 温箱中于 $37{ }^{\circ} \mathrm{C}$ 培养, $4 \mathrm{~d}$ 后弃去培养 液, 每孔加入 $200 \mu \mathrm{L} 0.2 \%$ MTT 溶液(RPMI1640 配制). 再在 $37{ }^{\circ} \mathrm{C}$ 下保温 $4 \mathrm{~h}$, 弃去上清液, 每孔加入 DMSO $150 \mu \mathrm{L}$ 溶解甲皆颗粒, 轻度振荡后, 用酶标仪. 在参考 波长 $450 \mathrm{~nm}$ 、检测波长 $570 \mathrm{~nm}$ 条件下测定光密度值 (OD). 以浓度为横轴, 细胞存活率为纵轴绘制曲线, 用 两点法(Reed and Muench 法)计算 $\mathrm{IC}_{50}$ 值, 结果见表 1.

表 1 不同化合物对两种肿瘤细胞株的半数抑制浓度 $\left[\mathrm{IC}_{50} /\left(\mu \mathrm{mol} \cdot \mathrm{L}^{-1}\right)\right]$

Table 1 Half-inhibitory concentration of different compounds to two tumor cell lines $\left[\mathrm{IC}_{50} /\left(\mu \mathrm{mol} \cdot \mathrm{L}^{-1}\right)\right]$

\begin{tabular}{ccc}
\hline Compound & HepG2 & HCT-116 \\
\hline GA & 2.9 & 2.8 \\
$\mathbf{5}$ & 1.1 & 2.3 \\
$\mathbf{7}$ & 1.1 & 1.9 \\
$\mathbf{8}$ & 1.7 & 2.0 \\
$\mathbf{9}$ & 3.3 & 5.4 \\
$\mathbf{1 0}$ & 16.5 & 4.8 \\
$\mathbf{1 1}$ & 3.8 & 3.9 \\
$\mathbf{1 2}$ & 6.5 & 4.1 \\
$\mathbf{1 3}$ & 2.7 & 1.5 \\
$\mathbf{1 4}$ & 7.7 & 3.7 \\
$\mathbf{1 5}$ & 6.4 & 1.8 \\
$\mathbf{1 6}$ & 6.5 & 1.4 \\
$\mathbf{1 7}$ & 1.9 & 3.8 \\
$\mathbf{1 8}$ & 4.9 & 1.5 \\
$\mathbf{1 9}$ & $>100$ & $>100$ \\
$\mathbf{2 0}$ & 10.3 & 5.5 \\
$\mathbf{2 1}$ & $>100$ & $>100$ \\
$\mathbf{2 2}$ & 23.4 & 21.9 \\
\hline
\end{tabular}

\section{2 结果与讨论}

本文以藤黄酸为原料, 经过 5 7 步反应合成了 17 个藤黄酸衍生物. 由于藤黄酸有多个烯键, 因此选择性 地在 32,33 位氧化是这条路线的关键. 间氯过氧苯甲酸 ( $m$-CPBA) 制备环氧化物 ${ }^{[10,11]}$ 是过酸对烯的亲电进攻过 程, 一般来讲烯烃分子中有给电子取代基能促进反应的 进行 $^{[12]}$. 此外, 间氯过氧苯甲酸进行环氧化反应时, 还
要考虑位阻效应, 就是试剂在进攻底物时, 底物上的取 代基与试剂之间的排斥作用而引起的区域选择性. 我们 实验证实以间氯过氧苯甲酸为环氧化剂的藤黄酸的环 氧化反应主要发生在 C-32, C-33 及 C-37, C-38 位烯键, 不发生在具有吸电子基团以及空间位阻的 C-27, C-28 和 C-9, C-10 位的烯键上, 并顺利得到化合物 2 与 C-32, C-33 及 C-37, C-38 位双环氧化的化合物(物质的量的比 为 $2: 3$ ).

文献[13]报道了由藤黄酸得到 36 位羧基和 6 位羟基 双甲基化的甲基藤黄酸的方法, 即将藤黄酸溶于丙酮 中, 再同时加入碳酸钾和碘甲烷, 反应需 1 周时间; 同 样, 我们采取这种方法用于化合物 $\mathbf{5}$ 的合成, 发现反应 时间依然需要 1 周, 于是我们对此反应进行了改进, 将 化合物 $\mathbf{1}$ 溶于丙酮后, 先将碳酸钾加入反应液中, 搅拌 $0.5 \mathrm{~h}$ 后再滴加碘甲烷，大大缩短了反应时间，仅用 $24 \mathrm{~h}$, 收率大于 $95 \%$.

在合成化合物 $10 \sim 14$ 的过程中, 由于在仲胺化合 物存在时, $\alpha, \beta$-不饱和酮易与仲胺发生迈克尔加成反 应 $^{[13]}$, 因此合成这类化合物时要避免该副反应的发生. 我们将化合物 5 溶于四氢呋喃中, 加入 EDCI 和 DMAP 搅拌 $0.5 \mathrm{~h}$ 后, 在冰盐浴条件下, 缓慢滴加等摩尔的仲胺 化合物的四氢呋喃溶液, 保持 $0{ }^{\circ} \mathrm{C}$ 反应 $8 \mathrm{~h}$, 得到目标 产物 10 14, 收率为 $23 \% \sim 36 \%$, TLC 检测仅发现极少 量的副产物生成.

在合成化合物 19 22 的过程中, 由于氨基酸参与 的反应一般涉及到氨基酸的保护与脱保护，我们考虑到 氨基酸脱保护时所用的酸性或碱性条件可能会导致藤 黄酸桥环结构的不稳定. 因此我们采用另一种策略, 将 化合物 5 溶于二氯甲烷中, 加入 DCC 活化后, 再加入 $N$-羊基琥珀酸亚胺, 得到化合物 7. 化合物 7 经粗分后, 溶于二甲基亚砜中, 再加入氨基酸和三乙胺, 顺利得到 目标产物 19 22, 收率为 23\% 33\%.

活性测试结果表明, 藤黄酸 C-32 位取代衍生物对 所篮的两株肿瘤细胞表现出较好的增殖抑制活性. 其中 化合物 5, 7, 8, 13, 17 对人肝癌细胞(HepG2)的增殖抑制 活性显著强于藤黄酸, 而化合物 $5,7,8,13,16,18$ 则对 人结肠癌细胞(HCT-116)的增殖抑制活性比藤黄酸更强. 藤黄酸化学结构中 C-32, C-33 双键切断引入羧基后, 羧 基修饰的一系列化合物中部分化合物抗肿瘤活性强于 阳性对照 GA 或相当, 说明 B 环上异戊烯基对于藤黄酸 的增殖抑制活性没有 $\mathrm{A}$ 环侧链重要，是可修饰的.

藤黄酸 C-32 位羧基成乙酯和异丙酯后, 也表现出 明显强于藤黄酸的抗肿瘤活性; 与芳胺成酰胺后, 对人 结肠癌细胞(HCT-116)的增殖抑制活性也明显加强, 说 明 C-32 位引入亲酯性基团能提高 $\mathrm{GA}$ 的生物活性. 而藤 
黄酸 C-32 位羧基接入亲水性胺或氨基酸后, 大部分化 合物均表现出弱于 GA 的生物活性, 说明 C-32 位引入亲 水性基团会得到相反的结果，降低其抗肿瘤活性.

\section{References}

[1] Yi, T.-F.; Yi, Z.-F.; Cho, S. G.; Luo, J.; Pandey, M. K.; Aggarwal, B. B.; Liu, M.-Y. Cancer Res. 2008, 68, 1843.

[2] Qin, Y.-X.; Meng, L.-H.; Hu, C.-X.; Duan, W.-H.; Zuo, Z.-L.; Lin, L.-P.; Zhang, X.-W.; Ding, J. Mol. Cancer Ther. 2007, 6, 2429.

[3] Gu, H.-Y.; Wang, X.-T.; Rao, S.-Y.; Wang, J.; Zhao, J.; Ren, F.-L.; Mu, R.; Yang, Y.; Qi, Q.; Liu, W.; Lu, N.; Ling, H.; You, Q.-D.; Guo, Q.-L. Mol. Cancer Ther. 2008, 7, 3298.

[4] Zhang, H.-Z.; Kasibhatla, S.; Wang, Y.; Herich, J.; Guastella, J.; Tseng, B.; Drewe, J.; Cai, S.-X. Bioorg. Med. Chem. 2004, 12, 309.

[5] He, L.-Q.; Ling, Y.; Fu, L.; Yin, D.-K.; Wang, X.-S.; Zhang, Y.-H.
Bioorg. Med. Chem. Lett. 2012, 22, 289.

[6] Feng, F.; Liu, W.-Y.; Chen, Y.-S.; Guo, Q.-L; You, Q.-D. J. China Pharm. Univ. 2005, 36, 302. (in Chinese)

(冯锋, 柳文媛, 陈优生, 郭青龙, 尤启冬, 中国药科大学学报, 2005, 36, 302.)

[7] Wang, J.-X.; Ma, J.-H.; You, Q.-D.; Zhao, L.; Wang, F.; Li, C.; Guo, Q.-L. Eur. J. Med. Chem. 2010, 45, 4343.

[8] Wang, J.-X.; Zhao, L.; Hu, Y.; Guo, Q.-L.; Zhang, L.; Wang, X.-J.; Li, N.-G.; You, Q.-D. Eur. J. Med. Chem. 2009, 44, 2611.

[9] Wang, X.-J.; Lu, N.; Yang, Q.; Dai, Q.-S.; Tao, L.; Guo, X.-K.; Guo, Q.-L.; You, Q.-D. Bioorg. Med. Chem. Lett. 2010, 20, 2438.

[10] Swern, D. Org. React. 1953, 7, 378.

[11] Anderson, W. K.; Veysoglu, T. J. Org. Chem. 1973, 38, 2267.

[12] Lee, J. B; Uff, B. C. Quar. Rev. 1967, 21, 429.

[13] Cai, S.-X.; Zhang, H.-Z.; Wang Y.; Tseng, B; Kasibhatla, S; Drewe, J. WO 0044216, 2000 [Chem. Abstr. 2000, 133, 150415]. 